Copyright (C1996, American Institute of Aeronautics and Astronautics, Inc.

AIAA Meeting Papers on Disc, 1996, pp. 1938-1950

A9626995, AIAA Paper 96-1545

\title{
Forced response of mistuned bladed disks using reduced-order modeling
}

\author{
Marlin Kruse \\ Michigan Univ., Ann Arbor \\ Christophe Pierre \\ Michigan Univ., Ann Arbor
}

\section{IN:AIAA/ASME/ASCE/AHS/ASC Structures, Structural Dynamics and Materials} Conference and Exhibit, 37th, Salt Lake City, UT, Apr. 15-17, 1996, Technical Papers. Pt. 4 (A96-26801 06-39), Reston, VA, American Institute of Aeronautics and Astronautics, 1996, p. 1938-1950

\begin{abstract}
A reduced-order model formulation is presented for examining the forced response of tuned and mistuned bladed disks. The technique developed uses modal information obtained from highly detailed FEM models to create, in a systematic manner, much simpler and computationally inexpensive models of bladed disks. The small size of the reduced-order model and associated computational savings enable analysts to examine the effect of mistuning strength and pattern, interblade coupling, and localized modes on forced response amplitudes. Previously, this was a formidable task with finite element modeling for even a single mistuning pattern. (Author)
\end{abstract}




\title{
FORCED RESPONSE OF MISTUNED BLADED DISKS USING REDUCED-ORDER MODELING
}

\author{
Marlin Kruse* and Christophe Pierre ${ }^{\dagger}$ \\ The University of Michigan, Ann Arbor, Michigan
}

\begin{abstract}
A reduced-order model formulation is presented for examining the forced response of tuned and mistuned bladed disks. The technique developed uses modal information obtained from highly detailed finite element models to create, in a systematic manner, much simpler and computationally inexpensive models of bladed disks. The small size of the reduced-order model and associated computational savings enable analysts to examine the effect of mistuning strength and pattern, interblade coupling, and localized modes on forced response amplitudes. Previously, this was a formidable task with finite element modeling for even a single mistuning pattern.
\end{abstract}

\section{Introduction}

Bladed disks are spatially repetitive structures, and their geometry is fully described by that of a fundamental region, or sector. In addition, the dynamic characteristics of a sector determine the dynamic behavior of the entire structure. Modern structural analysis of bladed disks takes advantage of this fact and is greatly simplified by the assumption of cyclic symmetry. A structural model of a generic bladed disk is illustrated in Figure 1. Cyclic symmetry enables engineers to create highly detailed finite element models of a single sector, shown in Figure 2, in order to examine the dynamic response of the entire assembly. However, cyclic symmetry implies that all sectors in a blade assembly are identical, that is, that the system is tuned. Unfortunately, small differences in the structural properties of individual blades, due to manufacturing and material tolerances, or in-service degradation, often destroy ideal symmetry. These irregularities are referred to as blade mistuning. The collapse of symmetry may lead to qualitatively different dynamic behavior than that experienced by a perfectly tuned assembly. In particular, irreqularities may inhibit the propagation of vibrations within the structure and confine the vibrational energy to mostly a single sector. Mode shapes may become spatially localized and, as a result, a single blade may experience deflections much larger than that predicted by a tuned analysis.

Copyright (C) 1996 by Marlin Jay Kruse. Published by the American Institute of Aeronautics and Astronautics, Inc. with permission.

* Graduate Student Research Assistant.

$\dagger$ Associate Professor, Mechanical Engineering and Applied Mechanics, Member AIAA.

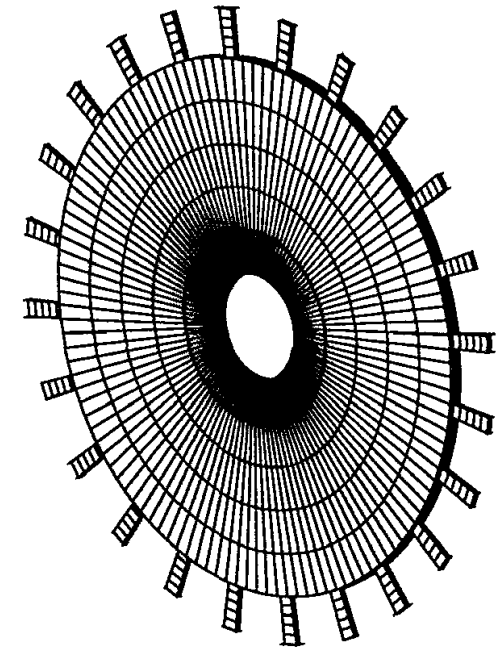

Figure 1: Full bladed-disk structural model.

Wei and Pierre ${ }^{1}$ documented the existence of localized modes in mistuned bladed disks through the investigation of a single-degree of freedom per sector model. Lumped-mass models of bladed disks, such as that used by Wei and Pierre, capture the basic dynamics of the assembly, but are difficult to relate to more descriptive finite element models. Correlation of lumped mass and finite element models requires a tedious iteration of mass and stiffness parameters until satisfactory correlation is established. In general, there is no organized or rigorous approach for developing lumped mass models from finite element models.

In practice, blade mistuning is random, and statistical analyses that utilize computational Monte Carlo simulations are critical in predicting the response amplitudes of bladed disks. A Monte Carlo 


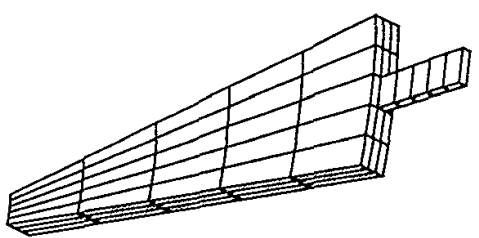

Figure 2: Single sector structural model.

simulation of a full finite element blade assembly, however, is enormously costly, and is not even feasible for most industrial turbomachinery rotors (see Kruse and Pierre ${ }^{2}$ ). Reduced-order modeling (ROM) is thus required in order to enable statistical analysis of bladed disk response. In 1994, Ottarsson et $a l^{3}{ }^{3}$ introduced a technique for developing ROM of bladed disks directly from finite element models. The procedure involves a component mode analysis of the bladed disk, with a truncated number of modal amplitudes describing the response of the assembly. The key idea introduced by Ottarsson et $a l^{3}$ is that the motion of an individual blade consists of both cantilever blade elastic motion and diskinduced static motion. The principal advantage of the technique is the considerable computational savings associated with solving a full mistuned rotor with a reduced set of degrees of freedom. A second advantage is that the ROMs can be systematically generated from finite element models. Since finite element models are used extensively in the design process, the method is likely to have an significant impact on actual turbomachinery designs.

In this paper, Ottarson et al.'s free response formulation for tuned and mistuned bladed disks is extended to the forced response case. The formulation of the forcing vector in the ROM generalized coordinates is described in Section 2. In Section 3, validation results are presented for a selected case study. The ROM is then used to examine the physical mechanisms of vibration localization and forced response amplitude increase due to mistuning. Finally, Section 4 contains a brief statistical study of forced response amplitudes using the ROM.

There are three significant contributions of this work. First, the technique presented establishes a systematic approach for developing ROMs that are representative of industrial turbomachinery rotors, and for obtaining their forced response in an accurate and inexpensive way. Second, the method will enable engineers to examine the effect of blade mistuning and interblade coupling on forced response amplitudes; this was not possible to date with finite element models. Third, it will also enable designers to carry out parametric studies of mistuned systems, and to determine the statistical variation in stress amplitudes due to random mistuning.

\section{Reduced-Order Model} Formulation for Forced Response

In the ROM formulation for bladed disk free response, Ottarsson et al. ${ }^{3}$ introduce the idea that the motion of an individual blade consists of cantilever blade elastic motion and disk-induced static motion. Figure 3 illustrates these two types of blade motion. The finite element displacements of the blades are thus expanded as:

$$
\mathbf{u}=\mathrm{U}^{\mathbf{d}} \mathbf{a}+\mathbf{U}^{\mathbf{b}} \mathbf{b}
$$

where $\mathbf{U}^{\mathbf{d}}$ is the blade portion of the disk-induced mode shapes, $\mathbf{U}^{\mathbf{b}}$ contains the mode shapes of the cantilevered blades, and $\mathbf{a}$ and $\mathbf{b}$ represent disk and blade modal coordinates, respectively.

In order to derive the ROM from the standard finite element model of a sector, two finite element models of bladed-disk components are required. The first model is that of a single disk-blade sector. Cyclic symmetry is utilized to determine the disk-induced static mode shapes, $\tilde{\mathbf{u}}_{\mathbf{n}, m}^{\mathrm{d}}$. Since static blade motion is sought, the blade elements are massless. The second model is that of a cantilevered blade, leading to the generation of the elastic cantilever blade mode shapes, $\tilde{\mathbf{u}}_{\mathbf{i}}^{\mathbf{b}}$.

The derivation of the ROM equations of free motion for tuned and mistuned bladed disks is detailed in Reference. ${ }^{3}$ The final form of the equations is restated here for convenience:

$$
\begin{aligned}
& {\left[\begin{array}{cc}
\mathbf{I}+\mathbf{B d i a g}\left[\tilde{\mathbf{U}}_{\mathbf{n}}^{\mathbf{d}^{\mathrm{T}}} \tilde{\mathbf{M}}_{\mathbf{b}} \tilde{\mathbf{U}}_{\mathbf{n}}^{\mathrm{d}}\right] & \mathbf{U}^{\mathbf{d}^{\mathrm{T}}} \mathbf{M}_{\mathrm{b}} \mathbf{U}^{\mathrm{b}} \\
\mathbf{U}^{\mathbf{b}^{\mathrm{T}}} \mathbf{M}_{\mathrm{b}} \mathbf{U}^{\mathbf{d}} & \mathbf{I}
\end{array}\right]\left[\begin{array}{c}
\ddot{a} \\
\ddot{\mathrm{b}}
\end{array}\right]} \\
& +\left[\begin{array}{cc}
\hat{\mathbf{K}}_{\mathbf{d}}+\left[\tilde{\mathbf{U}}_{\mathbf{n}}^{\mathbf{d}^{\mathrm{T}}} \tilde{\mathbf{K}}_{\mathbf{b}} \tilde{\mathbf{U}}_{\mathbf{n}}^{\mathrm{d}}\right] & \mathbf{U}^{\mathbf{d}^{\mathrm{T}}} \mathbf{K}_{\mathbf{b}} \mathbf{U}^{\mathbf{b}} \\
\mathbf{U}^{\mathbf{d}^{\mathrm{T}}} \mathbf{K}_{\mathbf{b}} \mathbf{U}^{\mathbf{b}} & \operatorname{diag}\left(1+\delta_{i}\right) \otimes \hat{\mathbf{K}}_{\mathbf{b}}
\end{array}\right]\left[\begin{array}{l}
\mathbf{a} \\
\mathbf{b}
\end{array}\right] \\
& =\left[\begin{array}{l}
0 \\
0
\end{array}\right]
\end{aligned}
$$

where Bdiag denotes a block diagonal matrix, and diag denotes a diagonal matrix.

In this section, the ROM is generalized to the case of external excitation, and the corresponding equations of forced motion are derived. Consider that the bladed disk is subject to an arbitrary external force vector, $F$, which may be applied to all the blade degrees of freedom of the assembly. The corresponding modal force vector is derived from Hamilton's Principle:

$$
\int_{t_{1}}^{t_{2}}(\delta U-\delta T) d t=\int_{t_{1}}^{t_{2}} \delta W_{e x t} d t
$$


where the external virtual work is

$$
\int_{t_{1}}^{t_{2}} \delta W_{e x t} d t=\int_{t_{1}}^{t_{2}} \delta \mathbf{u}^{\mathbf{T}} \mathbf{F} d t
$$

$\mathbf{u}$ is the displacement vector containing all blade degrees of freedom in the assembly and $\mathbf{F}$ is the corresponding force vector on those degrees of freedom. Substituting Eq. (1) into Eq. (4) yields

$$
\int_{t_{1}}^{t_{2}} \delta W_{e x t} d t=\int_{t_{1}}^{t_{2}} \delta \mathrm{b}^{\mathrm{T}} \mathbf{U}^{\mathrm{b}^{\mathrm{T}}} \mathbf{F} d t+\int_{t_{1}}^{t_{2}} \delta \mathbf{a}^{\mathrm{T}} \mathbf{U}^{\mathrm{d}^{\mathrm{T}}} \mathbf{F} d t
$$

from which the equations of forced motion are obtained as

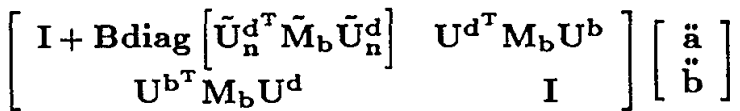

$$
\begin{aligned}
& +\left[\begin{array}{cc}
0 & 0 \\
0 & \operatorname{diag}(2 \zeta) \otimes \sqrt{\hat{\mathbf{K}}_{\mathrm{b}}}
\end{array}\right]\left[\begin{array}{c}
\dot{\mathbf{a}} \\
\dot{\mathbf{b}}
\end{array}\right]+\left(1+j G_{\text {Struc }}\right)
\end{aligned}
$$

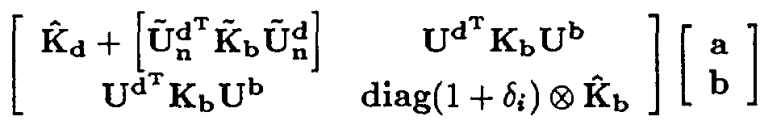

$$
\begin{aligned}
& =\left[\begin{array}{l}
\mathbf{U}^{\mathrm{d}^{\mathrm{T}}} \mathbf{F} \\
\mathbf{U}^{\mathbf{b}^{\mathrm{T}}} \mathbf{F}
\end{array}\right]
\end{aligned}
$$

Note that in addition to external excitation, both structural and viscous damping have been introduced into the ROM.

The external excitation is assumed to be harmonic in time and to differ only in phase from sector to sector, or blade to blade. This type of excitation results from the rotation of the bladed disk under a stationary force in the flow field, and has been widely used in the literature. ${ }^{4,5}$ The external force vector can then be expressed as

$$
\mathbf{F}=\left\{\begin{array}{c}
\tilde{\mathbf{f}} \\
\tilde{\mathbf{f}} e^{j \phi_{1}} \\
\vdots \\
\tilde{\mathbf{f}} e^{j \phi_{N-1}}
\end{array}\right\}
$$

where

$$
\phi_{i}=\frac{2 \pi C(i-1)}{N} \quad i=1, \ldots, N
$$

is the interblade phase angle of the excitation, $\tilde{\mathbf{f}}$ is the force vector on a single blade, and $C$ is the engine order excitation. The expression for the ROM force vector in Eq. 6, $\left[\begin{array}{lll}\mathbf{U}^{\mathbf{d}^{\mathbf{T}}} \mathbf{F} & \mathbf{U}^{\mathbf{b}^{\mathbf{T}}} \mathbf{F}\end{array}\right]^{T}$, is not practical because of the size of the quantities involved, which pertain to the entire assembly. A more convenient form that minimizes memory storage can be expressed in terms of the blade force vector, $\tilde{\mathbf{f}}$, and the disk-induced and cantilever blade mode shapes of a single sector, $\tilde{\mathbf{u}}_{\mathbf{n}, \mathbf{m}}^{\mathbf{d}}$ and $\tilde{\mathbf{u}}_{\mathbf{i}}^{\mathbf{b}}$, respectively. This formulation requires using the properties of circulant matrices (see Appendix A or the book by Davies ${ }^{6}$ ), as follows.

To begin, the disk portion of the modal force vector, $\mathbf{U}^{d^{T}} \mathbf{F}$, is expressed in terms of sector-referred quantities. The assembly force vector, $\mathbf{F}$, is first expressed in terms of the sector force vector as:

$$
\mathbf{F}=\sqrt{N} \mathbf{e}_{\mathbf{C}} \otimes \tilde{\mathbf{f}}
$$

where $\otimes$ is the Kronecker product and $e_{C}$ is the $C$ th column of the Fourier matrix, both defined in Appendix A. The disk-induced static mode shapes for the assembly, $\mathbf{U}^{\mathbf{d}}$, are then expressed in terms of those for the individual sectors as:

$$
\mathbf{U}^{\mathbf{d}}=\left[\mathbf{U}_{0}^{\mathrm{d}}, \ldots, \mathbf{U}_{\mathbf{n}}^{\mathrm{d}}, \ldots, \mathbf{U}_{\mathbf{P}}^{\mathrm{d}}\right]
$$

where

$$
\mathrm{U}_{\mathbf{n}}^{\mathrm{d}}=\quad \begin{aligned}
& {\left[\mathbf{e}_{\mathbf{n}} \otimes \tilde{\mathbf{u}}_{\mathbf{n}, \mathbf{1}}^{\mathrm{d}}, \mathbf{e}_{\mathbf{n}}^{*} \otimes \tilde{\mathbf{u}}_{\mathbf{n}, 1}^{\mathrm{d}^{*}}, \ldots\right.} \\
& \left.\ldots, \mathbf{e}_{\mathbf{n}} \otimes \tilde{\mathbf{u}}_{\mathbf{n}, \mathbf{m}_{\mathbf{d}}}^{\mathrm{d}}, \mathbf{e}_{\mathbf{n}}^{*} \otimes \tilde{\mathbf{u}}_{\mathbf{n}, \mathbf{m}_{d}}^{\mathrm{d}^{*}}\right]
\end{aligned}
$$

and ${ }^{*}$ denotes the complex conjugate. Note that the 0 th and, for $N$ even, the $P$ th harmonic modes do not occur in complex conjugate pairs. Using Eqs. (9) and (11), $\mathbf{U}_{\mathbf{n}}^{\mathbf{d}^{\mathbf{T}}} \mathbf{F}$ is expressed as:

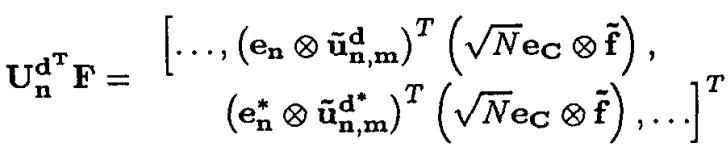

Taking the transpose and making use of Eq. (A.5) yields:

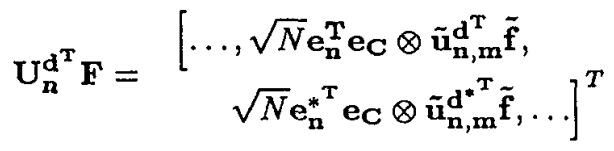

The Fourier columns, $\mathbf{e}_{\mathbf{n}}$ and $\mathbf{e}_{\mathbf{n}}^{*}$, are orthogonal to ec except when $n=C$. Thus, the engine order excitation, $C$, determines which mode shapes of the assembly are being excited. The disk portion of the modal force vector, $\mathbf{U}^{\mathrm{d}^{T}} \mathbf{F}$, is zero except for the $C$ harmonic mode shapes. This can be restated as

$$
\mathbf{U}^{\mathbf{d}^{\mathrm{T}}} \mathbf{F}=\left[0, \ldots, 0,\left(\mathbf{U}_{\mathbf{C}}^{\mathbf{d}^{\mathrm{T}} \mathbf{F}}\right)^{T}, 0, \ldots, 0\right]^{T}
$$

Using the cyclic symmetry property, ${ }^{3}$ a finite element analysis typically yields the real and imaginary 
(cosinus and sinus) parts of the modes of a sector. Hence, the disk-induced static blade modes are written as:

$$
\tilde{\mathbf{u}}_{\mathbf{n}, \mathbf{m}}^{\mathrm{d}}, \tilde{\mathbf{u}}_{\mathbf{n}, \mathbf{m}}^{\mathrm{d}^{*}}=\tilde{\mathbf{u}}_{\mathbf{n}, \mathbf{m}}^{\mathrm{c}} \pm j \tilde{\mathbf{u}}_{\mathbf{n}, \mathbf{m}}^{\mathbf{s}}
$$

The corresponding pair of complex conjugate modes for the entire assembly are generated from the Kronecker product of the $n$th harmonic column of the Fourier matrix and the complex conjugate pair of disk-induced modes are:

$$
\begin{aligned}
& \mathbf{U}_{\mathbf{n}, \mathbf{m}}^{\mathbf{d}}=\mathbf{e}_{\mathbf{n}} \otimes\left(\tilde{\mathbf{u}}_{\mathbf{n}, \mathbf{m}}^{\mathrm{c}}+j \tilde{\mathbf{u}}_{\mathbf{n}, \mathbf{m}}^{\mathbf{s}}\right) \\
& \mathbf{U}_{\mathbf{n}, \mathbf{m}}^{\mathbf{d}^{*}}=\mathbf{e}_{\mathbf{n}}^{*} \otimes\left(\tilde{\mathbf{u}}_{\mathbf{n}, \mathbf{m}}^{\mathrm{c}}-j \tilde{\mathbf{u}}_{\mathbf{n}, \mathbf{m}}^{\mathbf{s}}\right)
\end{aligned}
$$

We may rewrite Eq. (16) as:

$$
\begin{aligned}
& \mathrm{U}_{\mathbf{n}, \mathbf{m}}^{\mathrm{d}}=\left(\underline{\mathrm{e}}_{\mathbf{n}}^{\mathrm{c}}+j \underline{\mathrm{e}}_{\mathbf{n}}^{\mathrm{s}}\right) \otimes\left(\tilde{\mathbf{u}}_{\mathbf{n}, \mathbf{m}}^{\mathbf{c}}+j \tilde{\mathbf{u}}_{\mathbf{n}, \mathbf{m}}^{\mathrm{s}}\right) \\
& \mathrm{U}_{\mathrm{n}, \mathrm{m}}^{\mathrm{d}^{*}}=\left(\underline{\mathrm{e}}_{\mathrm{n}}^{\mathrm{c}}-j \underline{\mathrm{e}}_{\mathrm{n}}^{\mathrm{s}}\right) \otimes\left(\tilde{\mathrm{u}}_{\mathrm{n}, \mathrm{m}}^{\mathrm{c}}-j \tilde{\mathrm{u}}_{\mathrm{n}, \mathrm{m}}^{\mathrm{s}}\right)
\end{aligned}
$$

where $\underline{\underline{e}}_{n}^{\mathbf{c}}$ and $\underline{\mathbf{e}}_{\mathbf{n}}^{\mathbf{s}}$ denote the $n$th harmonic cosine and sine vectors from the real form of the Fourier matrix, Eq. (A.3). Note that $\underline{e}_{\mathbf{n}}^{\mathbf{c}}$ and $\underline{e}_{\mathbf{n}}^{\mathbf{s}}$ are essentially the real and imaginary portions of the complex form of the Fourier matrix, scaled to maintain unit modal masses for the real representation of the disk modes. This becomes, after simplification:

$$
\begin{aligned}
\mathbf{U}_{\mathbf{n}, \mathbf{m}}^{\mathbf{d}}, & \mathbf{U}_{\mathbf{n}, \mathbf{m}}^{\mathbf{d}^{*}}=\left[\underline{\mathbf{e}}_{\mathbf{n}}^{\mathbf{c}} \otimes \tilde{\mathbf{u}}_{\mathbf{n}, \mathbf{m}}^{\mathbf{c}}-\underline{\mathbf{e}}_{\mathbf{n}}^{\mathbf{s}} \otimes \tilde{\mathbf{u}}_{\mathbf{n}, \mathbf{m}}^{\mathrm{s}}\right] \\
& \pm j\left[\underline{\mathbf{e}}_{\mathbf{n}}^{\mathbf{s}} \otimes \tilde{\mathbf{u}}_{\mathbf{n}, \mathbf{m}}^{\mathbf{c}}+\underline{\mathbf{e}}_{\mathbf{n}}^{\mathbf{c}} \otimes \tilde{\mathbf{u}}_{\mathbf{n}, \mathbf{m}}^{\mathbf{s}}\right]
\end{aligned}
$$

Defining a new pair of eigenvectors as the real and imaginary portions of $\mathbf{U}_{\mathbf{n}, \mathbf{m}}^{\mathbf{d}}$ and $\mathbf{U}_{\mathbf{n}, \mathbf{m}}^{\mathbf{d}^{*}}$ yields, from Eqs. (12), (14), and (18):

$\mathrm{U}_{\mathrm{C}}^{\mathrm{d}^{\mathrm{T}}} \mathbf{F}=$

$$
\begin{aligned}
& {\left[\ldots,\left(\underline{\mathbf{e}}_{\mathbf{C}}^{\mathbf{c}} \otimes \tilde{\mathbf{u}}_{\mathbf{C}, \mathbf{m}}^{\mathbf{c}}-\underline{\mathbf{e}}_{\mathbf{C}}^{\mathbf{s}} \otimes \tilde{\mathbf{u}}_{\mathbf{C}, \mathbf{m}}^{\mathbf{s}}\right)^{T}\left(\sqrt{N} \mathbf{e}_{\mathbf{C}} \otimes \tilde{\mathbf{f}}\right)\right.} \\
& \left.\left(\underline{\underline{e}}_{\mathbf{C}}^{\mathbf{s}} \otimes \tilde{\mathbf{u}}_{\mathbf{C}, \mathbf{m}}^{\mathbf{c}}+\underline{\mathbf{e}}_{\mathbf{C}}^{\mathbf{c}} \otimes \tilde{\mathbf{u}}_{\mathbf{C}, \mathbf{m}}^{\mathbf{s}}\right)^{T}\left(\sqrt{N} \mathbf{e}_{\mathbf{C}} \otimes \tilde{\mathbf{f}}\right), \ldots\right]^{T}
\end{aligned}
$$

Taking the transpose and making use of Eq. (A.5) gives:

$$
\begin{aligned}
& \mathrm{U}_{\mathrm{C}}^{\mathrm{d}^{\mathrm{T}}} \mathrm{F}= \\
& {\left[\ldots, \sqrt{N} \underline{e}_{C}^{c^{\mathbf{T}}} \mathbf{e}_{\mathbf{C}} \otimes \tilde{\mathbf{u}}_{C, m}^{c^{\mathbf{T}}} \tilde{\mathbf{f}}-\sqrt{N} \underline{\mathbf{e}}_{\mathbf{C}}^{\mathbf{s}^{\mathbf{T}}} \mathbf{e}_{\mathbf{C}} \otimes \tilde{\mathbf{u}}_{\mathbf{C}, \mathbf{m}}^{\mathbf{s}^{\mathbf{T}}} \tilde{\mathbf{f}},\right.} \\
& \left.\sqrt{N} \underline{\mathbf{e}}_{\mathbf{C}}^{\mathbf{s}^{\mathbf{T}}} \mathbf{e}_{\mathbf{C}} \otimes \tilde{\mathbf{u}}_{\mathbf{C}, \mathbf{m}}^{\mathbf{c}^{\mathbf{T}}} \tilde{\mathbf{f}}+\sqrt{N} \underline{\mathbf{e}}_{\mathbf{C}}^{\mathbf{c}^{\mathrm{T}}} \mathbf{e}_{\mathbf{C}} \otimes \tilde{\mathbf{u}}_{\mathbf{C}, \mathbf{m}}^{\mathbf{s}^{\mathbf{T}}} \tilde{\mathbf{f}}, \ldots\right]^{T}
\end{aligned}
$$

Likewise, the blade portion of the modal forcing vector is

$$
\mathbf{U}^{\mathbf{b}^{\mathrm{T}}} \mathbf{F}=\left(\mathbf{I} \otimes \tilde{\mathbf{U}}^{\mathbf{b}}\right)^{T}\left[\left(\sqrt{N} \mathrm{e}_{\mathrm{C}}\right) \otimes \tilde{\mathbf{f}}\right]
$$

Taking the transpose and making use of Eq. (A.5) yields:

$$
\mathbf{U}^{\mathrm{b}^{\mathrm{T}}} \mathbf{F}=\left(\sqrt{N} \mathrm{e}_{\mathbf{C}}\right) \otimes\left(\tilde{\mathbf{U}}^{\mathrm{b}^{\mathrm{T}}} \tilde{\mathbf{f}}\right)
$$

The final form of the modal forcing vector, $\left[\begin{array}{c}\mathbf{U}^{\mathbf{d}^{\mathbf{T}} \mathbf{F}} \\ --\overline{\mathbf{U}^{\mathbf{T}} \mathbf{F}}\end{array}\right]$, is:

$$
\begin{gathered}
\left\{\begin{array}{c}
0_{1,1} \\
\vdots \\
0_{1, m_{d}}
\end{array}\right\} \\
\vdots \\
\left\{\begin{array}{c}
0_{C-1,1} \\
0_{C-1,1}^{*} \\
\vdots \\
0_{C-1, m_{d}} \\
0_{C-1, m_{d}}^{*}
\end{array}\right\}
\end{gathered}
$$

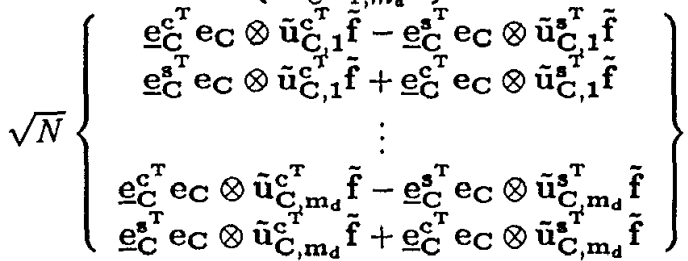$$
\left\{\begin{array}{c}
0_{C+1,1} \\
0_{C+1,1}^{*} \\
\vdots \\
0_{C+1, m_{d}} \\
0_{C+1, m_{d}}^{*}
\end{array}\right\}
$$

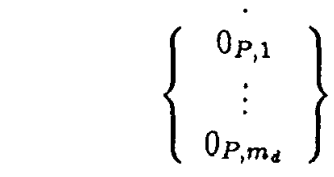

$$
\begin{aligned}
& \left\{\begin{array}{c}
e^{j 0} \tilde{\mathbf{u}}_{1}^{\mathbf{b}^{\mathbf{T}}} \tilde{\mathbf{f}} \\
\vdots \\
e^{j 0} \tilde{\mathbf{u}}_{\mathrm{m}_{\mathrm{b}}}^{\mathbf{b}_{\mathbf{T}}} \tilde{\mathbf{f}}
\end{array}\right\} \\
& \left\{\begin{array}{c}
e^{j \frac{3 \pi C}{N}} \tilde{\mathbf{u}}_{\mathbf{b}}^{b^{T}} \tilde{\mathbf{f}} \\
\vdots \\
e^{j \frac{2 \pi C}{N} \tilde{\mathbf{u}}_{\mathbf{m}_{\mathbf{b}}}^{\mathbf{b}^{\mathrm{T}}} \tilde{\mathbf{f}}}
\end{array}\right\} \\
& \left\{\begin{array}{c}
e^{j \frac{2 \pi(N-1) C}{N}} \tilde{\mathbf{u}}_{1}^{\mathbf{b}^{\mathrm{T}} \tilde{\mathbf{f}}} \\
\vdots \\
e^{j \frac{2 \pi(N-1) C}{N}} \tilde{\mathbf{u}}_{\mathbf{m}_{\mathrm{b}}}^{\mathbf{b}^{\mathrm{T}}} \tilde{\mathbf{f}}
\end{array}\right\}
\end{aligned}
$$

The principal advantage of expressing the modal forcing vector on a sector basis lies in the memory savings associated with storing the disk-induced and cantilever blade mode shapes of a sector, rather than the mode shapes for the entire assembly. In this manner, the sector mode shapes are projected onto 1941 


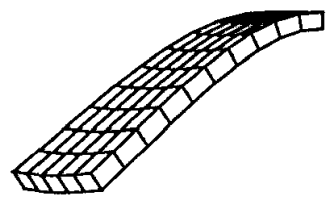

(a)

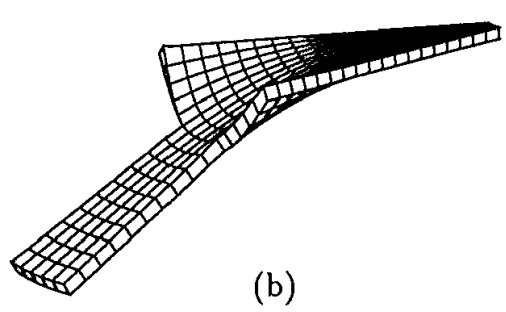

Figure 3: Cantilever blade (a) and disk-induced (b) motions.

the sector force vector to obtain modal forces. Cyclic symmetry arguments are then used to calculate the reduced-order model force vector. Thus, the ROM mass and stiffness matrices and the force vector are computed only once and then stored.

\section{Case Study of ROM Versus Finite Element Correlation}

Figure 2 depicts a single sector of a simplified bladed-disk with 24 blades. Table 1 lists material properties for the bladed disk. As an example and validation case, finite element and ROM forced responses are examined for both tuned and mistuned configurations. Cyclic symmetry routines in MSC/NASTRAN are used to calculate the forced response of the tuned finite element model. This model consists of eight-noded brick elements, with the inner hub of the disk constrained to have zero displacement. The disk portion of the model contains 75 elements, while the blade is modeled with five elements. There are 360 degrees of freedom per sector in the finite element model. In contrast, the reducedorder model consists of five disk-induced modes and four cantilever blade modes, for a total of nine degrees of freedom per sector.

The mistuned finite element model consists of the entire blade assembly, as shown in Figure 1. The same mesh pattern is used in the single-sector cyclic symmetry model and the full mistuned finite element model. Mistuning is introduced into the assembly by allowing each blade to have a different Young's modulus. For the ith blade, one has

$$
E_{i}=E_{o}\left(1+\delta_{i}\right)
$$

where $E_{o}$ is Young's modulus for a tuned blade, and $\delta_{i}$ is the dimensionless random mistuning, obtained from a uniform distribution with standard deviation $\sigma$. Table 2 lists the mistuning distribution used in the case study. Before proceeding with the forced response correlation, it will prove valuable to review the free response characteristics of the system.

\begin{tabular}{||c|c||}
\hline \hline Material Property & Property Value \\
\hline Modulus of Elasticity, $E_{o}$ & $200 \mathrm{GPa}$ \\
Modulus of Rigidity, $G$ & $80 \mathrm{GPa}$ \\
Density, $\rho$ & $7.86 \mathrm{Mg} / \mathrm{m}^{3}$ \\
Structural Damping, $G_{S t r u c}$ & 0.0025 \\
\hline \hline
\end{tabular}

Table 1: Bladed disk material properties.

\subsection{Free Response Characteristics}

It is convenient to describe the mode shapes of bladed disks in terms of nodal diameters and nodal circles. Nodal diameters are nodal lines across the diameter of the disk, while nodal circles are nodal lines in the circumferential direction. Information about the nodal diameter and nodal circle characteristics of a mode are conveniently summarized by a plot of natural frequencies versus the number of nodal diameters, as shown in Figure 4. Natural frequencies associated with zero nodal diameter are characterized by mode shapes in which all sectors vibrate in phase, while one-nodal diameter modes exhibit a $15^{\circ}$ phase shift between adjacent blades for this 24-blade assembly. The phase shift is incremented by $15^{\circ}$ for each consecutive increase in the number of nodal diameters, until each blade eventually vibrates $180^{\circ}$ out of phase with its nearest neighbors, corresponding to the twelve-nodal diameter case. Figure 5, for instance, illustrates a sixnodal diameter mode, with a $90^{\circ}$ phase shift between adjacent blades. For a general $N$-blade system, the phase shift between adjacent blades is

$$
\phi_{i}=\frac{2 \pi N_{d i a}(i-1)}{N} \quad i=1, \ldots, N
$$

where $\phi_{i}$ is the interblade phase angle, $N$ is the number of blades, and $N_{d i a}$ is the number of nodal diameters. These modes are accordingly referred to as constant interblade phase angle modes. Plotting the natural frequency versus the number of nodal diameters for the assembly in Fig. 1 reveals several interesting features. First, as the number of nodal diameters increases, the disk becomes stiffer rapidly. Thus, the slanted lines in Fig. 4 correspond to diskdominated modes. In the absence of blade tip or mid-span shrouding, the number of nodal diame- 


\begin{tabular}{||c|c||}
\hline \hline Blade Number & Mistuning $\delta_{i}$ \\
\hline 1 & -0.005173 \\
2 & -0.018767 \\
3 & -0.018214 \\
4 & -0.003899 \\
5 & -0.050068 \\
6 & -0.008520 \\
7 & 0.014171 \\
8 & 0.076210 \\
9 & 0.029285 \\
10 & 0.027175 \\
11 & 0.027682 \\
12 & -0.049229 \\
13 & -0.080759 \\
14 & -0.049068 \\
15 & 0.059333 \\
16 & -0.069234 \\
17 & -0.004147 \\
18 & 0.004288 \\
19 & 0.018403 \\
20 & -0.054743 \\
21 & 0.023942 \\
22 & 0.038109 \\
23 & 0.041102 \\
24 & 0.039275 \\
\hline \hline
\end{tabular}

Table 2: Random mistuning pattern, obtained from a uniform distribution with standard deviation $\sigma=$ $5 \%$.

ters does not significantly stiffen blade-dominated modes. Lines that are approximately horizontal, therefore, represent families of blade-dominated modes Second, there are six areas, called eigenvalue veerings, in which blade and disk families of modes veer away from each other. Physically, eigenvalue veerings are indicative of the degree of coupling between families of disk and blade modes. The strength of a veering is measured by the distance between the natural frequencies and the local curvature in the veering region. ${ }^{7}$

The nodal diameter description assumes that the mode shapes of the rotor are themselves cyclic, that is, spatially extended. This is true for tuned bladed disks. However, small blade mistuning may alter the mode shapes and cause the concentration of vibrational energy to mostly a single blade-the socalled phenomenon of localization. The observation that the first-order mode shape perturbation due to mistuning is inversely proportional to the difference in the tuned system's natural frequencies, leads to the well-known property that the localization of the mode shapes is most accute in frequency regions of

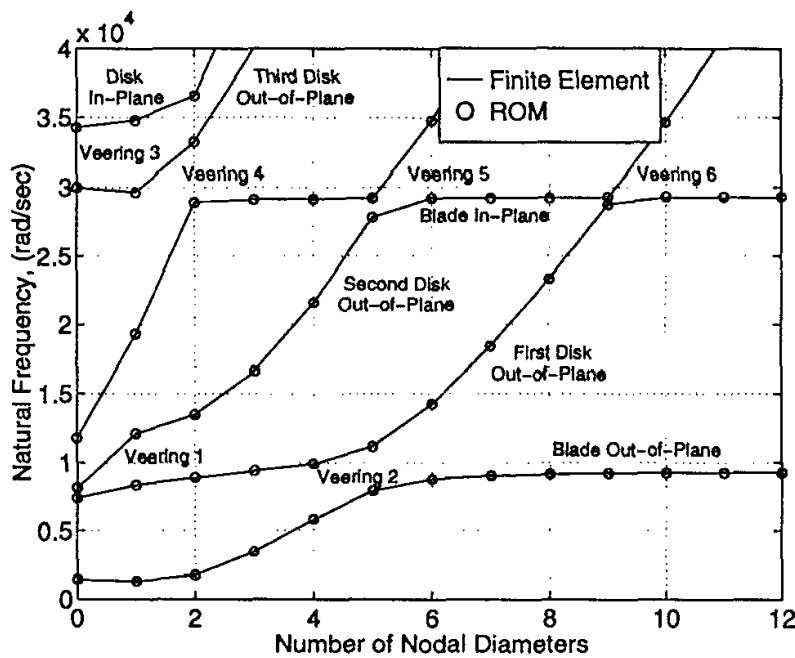

Figure 4: Natural frequencies versus number of nodal diameters. Note the excellent agreement between the finite element and the ROM natural fre-

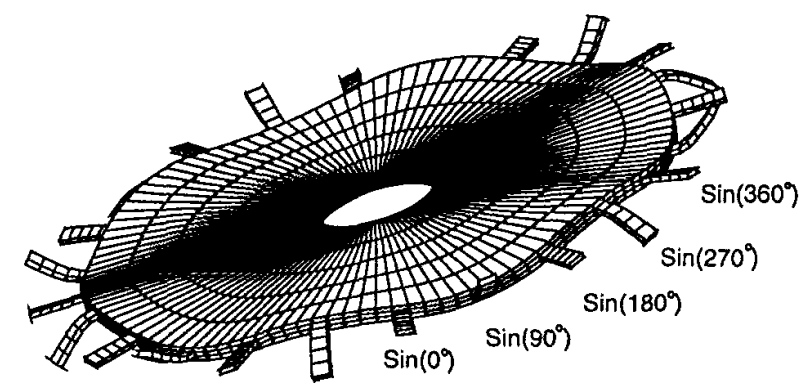

Figure 5: Six nodal diameter mode.

high modal density. ${ }^{7}$

From Fig. 4, it appears that both families of out-of-plane and in-plane blade modes exhibit high modal density and are therefore susceptible to mode localization upon the introduction of mistuning (here in-plane refers to the plane of the disk). Since the modal density for the in-plane modes is larger than that for the out-of-plane modes, localization effects are expected to be more prominent in the former. This was indeed observed in the study by Ottarsson et al. ${ }^{3}$ When mode localization occurs, vibrational energy is largely concencentrated in a single blade instead of being spatially extended throughout the structure; hence there is a strong likelihood that the forced response of the mistuned structure will greatly exceed the tuned response. ${ }^{8}$ 


\subsection{Forced Response: Out-of-Plane Loading}

Although typical external excitations consist of both in-plane and out-of-plane loading, these two types will be considered separately. Out-of-plane loading consists of right- and left-side harmonic unit loads located at the tip of the blade and normal to plane of the blisk. The engine order excitation, $C$, is equal to five. Thus, excitation varies in phase from blade to blade by 75 degrees. In terms of the nodal diameter description of the modes given earlier, this loading only excites the fifth nodal diameter out-ofplane blade mode of a tuned blisk. Note that the fifth engine order excitation lies in the middle of a strong eigenvalue veering (veering 2 in Fig. 4), between the first family of blade modes and the first family of disk modes. This particular excitation was found to be more susceptible to localization effects and to yield larger forced response amplitude increases due to mistuning than any of the other engine order excitations in this frequency range.

A scalar representation of blade deflection amplitude, the displacement norm, is utilized to represent the forced response of the assembly in terms of frequency. The displacement norm is defined as the square root of the sum of the squares of the blade displacement amplitudes for all blade degrees of freedom. In the tuned system response, all blades have identical displacement norms. This is not true, however, for a mistuned rotor, where all blades feature different response amplitudes. In the mistuned case, the largest displacement amplitude norm throughout the assembly is selected at each frequency, defining the largest frequency response.

Figure 6 depicts the frequency response of the tuned assembly using both finite element and reducedorder models, in the frequency region encompassing the first family of blade modes. It shows that the amplitude of the ROM resonant response, using the standard formulation given in Section 2, is within $7 \%$ of the finite element resonant response. Note the modest frequency offset of approximately $1 \%$. This offset can be largely eliminated with an eigenvalue adjustment for the cantilevered blade natural frequency of the out-of-plane blade mode. ${ }^{3}$ Note that the eigenvalue adjustment consists of an iteration of a single variable until nearly exact frequency correlation is obtained in Figs. 4 and 6 . With eigenvalue adjustment, the ROM resonant response only differs from the finite element response by $1 \%$. Figure 7 depicts a comparison of the mistuned finite element and ROM frequency responses. Again, the amplitude and frequency errors remain unchanged at $7 \%$ and $1 \%$, respectively, in the standard ROM form-

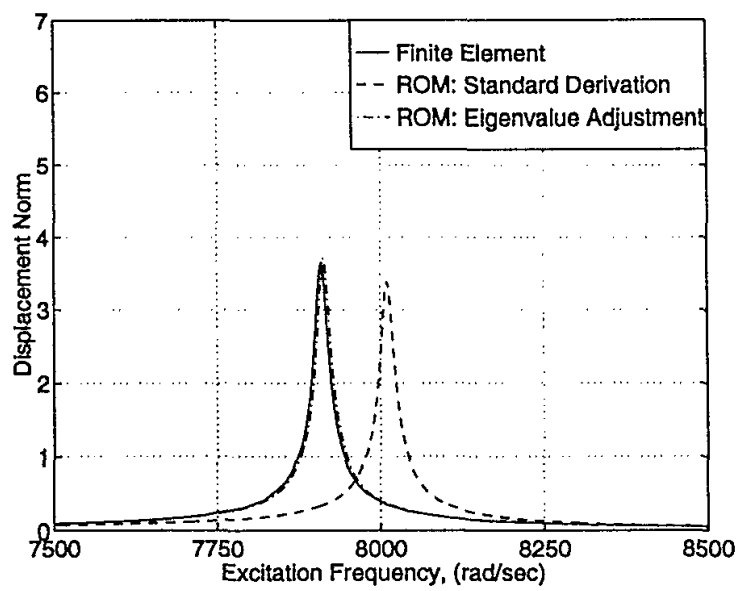

Figure 6: Comparison of tuned finite element and ROM frequency responses, for out-of-plane excitation with $C=5$.

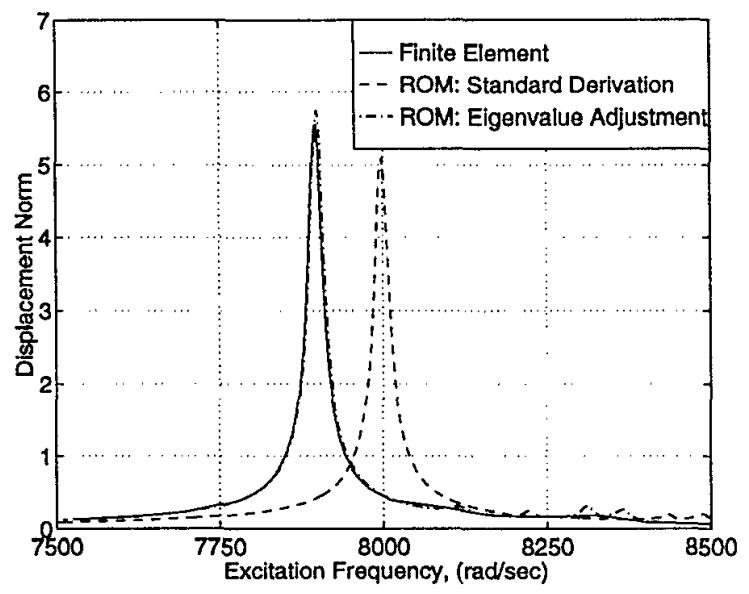

Figure 7: Comparison of mistuned finite element and ROM largest blade frequency responses, for outof-plane excitation with $C=5$.

\begin{tabular}{||c|c|c|c||}
\hline \hline & $\begin{array}{c}\text { Finite } \\
\text { Element }\end{array}$ & $\begin{array}{c}\text { ROM: } \\
\text { Standard } \\
\text { Derivation }\end{array}$ & $\begin{array}{c}\text { ROM: } \\
\text { Eigenvalue } \\
\text { Adjustment }\end{array}$ \\
\hline \multirow{3}{*}{ Tuned } & 375.1 & $357.9(-5 \%)$ & $405.6(+8 \%)$ \\
& -1.1 & $-1.0(-9 \%)$ & $-1.1(0 \%)$ \\
& -61.3 & $-59.3(-3 \%)$ & $-67.5(+10 \%)$ \\
\hline \multirow{3}{*}{ Mistuned } & 571.2 & $539.3(-6 \%)$ & $624.7(+9 \%)$ \\
& -1.6 & $-1.5(-8 \%)$ & $-1.8(+10 \%)$ \\
& -95.2 & $-90.9(-4 \%)$ & $-105.8(+11 \%)$ \\
\hline
\end{tabular}

Table 3: Comparison of finite element and ROM principal stresses $(\mathrm{MPa})$ at the resonant peak, for out-of-plane excitation with $C=5$. Principal stresses for the mistuned system can be as much as $52 \%$ higher than those for the tuned system. 
ulation. Adjusting the first out-of-plane blade natural frequency results in nearly exact amplitude and frequency correlations. Note in Figs. 6 and 7 that the increase in the largest resonant response amplitude due to mistuning is $55 \%$.

Once the displacement field has been solved for with the efficient ROM, displacements can be imported back into the finite element model for post processing of stress contours. Since all nodal displacements are prescribed, the finite element software merely performs the forward matrix calculations to obtain the stress field. Table 3 compares the resonant principal stresses obtained by finite element and reduced-order modeling. Principal stress values are listed for the first row of nodes past the root of the blade. Stress values at the root of the blade are not reported, since there is no fillet to alleviate the stress singularity. Principal stresses from the standard ROM formulation are 3 to $9 \%$ lower than the finite element model stresses, while principal stresses from the modified ROM (with eigenvalue adjustment) are 0 to $11 \%$ higher than the finite element stresses. The reader should note that the principal stresses for the mistuned model are as much as $52 \%$ larger than the tuned principal stresses. This $52 \%$ increase in principal stress corresponds to the $55 \%$ increase in resonant response amplitude observed from Figs. 6 to 7 .

\subsection{Forced Response: In-Plane Loading}

The in-plane loading consists of top and bottom harmonic unit loads located at the tip of the blade and acting in the circumferential direction. Here, the frequency range lies near the family of first inplane blade modes, and the engine order excitation is taken equal to zero. Thus, all blades in the assembly are subjected to identical, in phase loads. For a tuned system, it means that the zero nodal diameter, in-plane blade mode will be excited. Referring to Figure 4, eigenvalue veering 3 is characterized by a large separation distance and is significantly weaker than veering 2 in the out-of-plane case. Tuned results, depicted in Figure 8 , indicate a $10 \%$ resonant amplitude discrepancy between the finite element and standard ROM results. The frequency discrepancy, moreover, equals $6 \%$ with inplane loading, compared to $1 \%$ in the out-of-plane case (Fig. 6). Modifying the standard formulation by adjusting the eigenvalue associated with the first in-plane cantilevered blade mode results in nearly exact amplitude and frequency correlations for the forced response. Figure 9 illustrates the mistuned finite element and ROM largest frequency responses. Amplitude and frequency errors remain unchanged

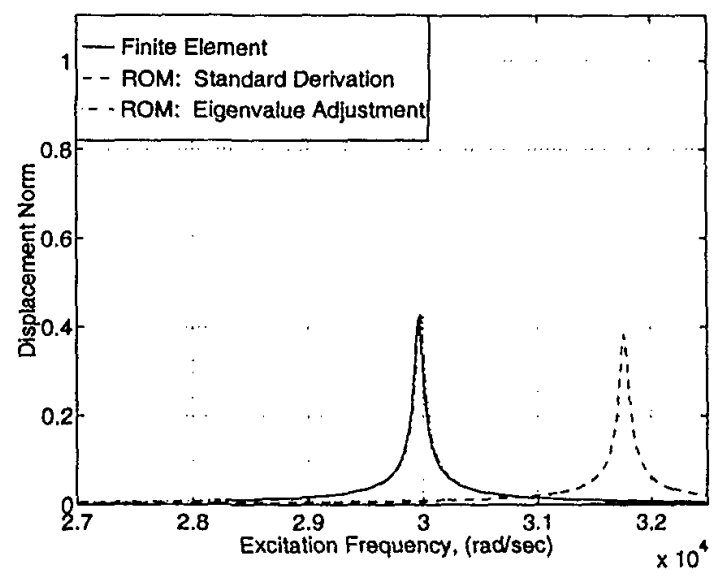

Figure 8: Comparison of tuned finite element and ROM frequency responses, for in-plane excitation with $C=0$.

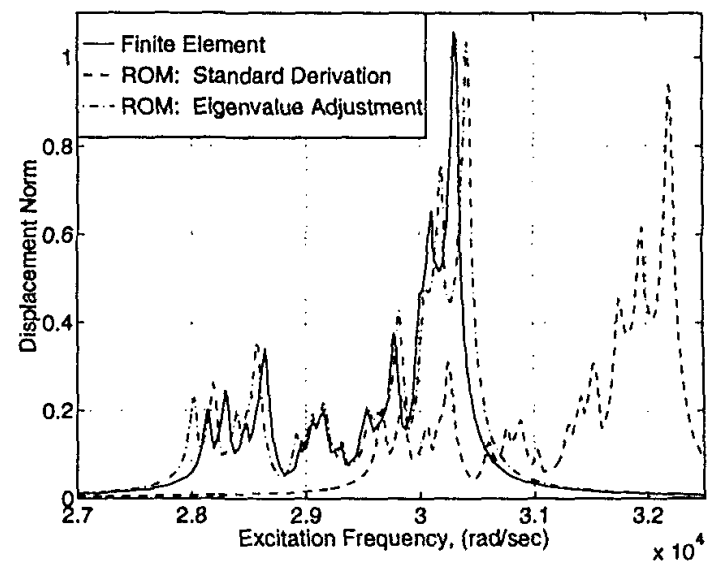

Figure 9: Comparison of mistuned finite element and ROM largest blade frequency responses, for inplane excitation with $C=0$.

\begin{tabular}{||c|c|c|c||}
\hline \hline & $\begin{array}{c}\text { Finite } \\
\text { Element }\end{array}$ & $\begin{array}{c}\text { ROM: } \\
\text { Standard } \\
\text { Derivation }\end{array}$ & $\begin{array}{c}\text { ROM: } \\
\text { Eigenvalue } \\
\text { Adjustment }\end{array}$ \\
\hline \multirow{3}{*}{ Tuned } & 182.7 & $186.8(+2 \%)$ & $209.4(+15 \%)$ \\
& -3.6 & $-3.7(+3 \%)$ & $-4.1(+14 \%)$ \\
& -30.8 & $-34.1(+11 \%)$ & $-38.2(+24 \%)$ \\
\hline \multirow{3}{*}{ Mistuned } & 480.4 & $475.7(-1 \%)$ & $528.0(+10 \%)$ \\
& -9.8 & $-9.4(-4 \%)$ & $-10.4(+6 \%)$ \\
& -81.2 & $-86.8(+7 \%)$ & $-96.3(+19 \%)$ \\
\hline
\end{tabular}

Table 4: Comparison of finite element and ROM principal stresses $(\mathrm{MPa})$ at the largest resonant peak, for in-plane excitation with $C=0$. Principal stresses for the mistuned system can be as much as $163 \%$ higher than stresses for the tuned system. 
at $10 \%$ and $6 \%$, respectively, in the standard formulation. Adjusting the first in-plane blade natural frequency results in nearly exact amplitude and frequency correlations. Figures 8 and 9 show that the increase in resonant response amplitude due to mistuning is $142 \%$.

Table 4 compares finite element and ROM principal stresses at resonance (in the mistuned case, the largest peak is selected). Principal stresses from the standard ROM formulation are from $4 \%$ lower to $11 \%$ higher than the finite element principal stresses. Principal stresses from the modified ROM are $6 \%$ to $24 \%$ higher than the finite element stresses. The resonant principal stresses for the mistuned assembly exceed the tuned principal stresses by as much as $163 \%$.

\subsection{Localization Phenomena and Structural Interblade Coupling}

It was shown above that the principal stress increase due to mistuning dramatically increased from $52 \%$ for out-of-plane excitation with $C=5$, to $163 \%$ for in-plane excitation with $C=0$. Previous sections have also alluded to eigenvalue veerings and how local curvature and separation distance between families of blade and disk modes are an indication of the coupling between these modes. In this section, we examine more closely the effect of both engine order excitation and eigenvalue veerings on the forced response of the case study rotor.

Although the high modal density associated with the blade modes indicates when localized modes may occur, the existence of localized modes alone is not enough to guarantee that the forced response amplitudes of a mistuned assembly will deviate significantly from those of a tuned system. Wei and Pierre $^{8}$ and Ottarsson and Pierre ${ }^{9}$ determined, for a single-degree of freedom per sector bladed disk model, that moderately weak coupling between blades is required for significant amplitude increases in the forced response. If there is no interblade coupling, then each blade acts as an individual mistuned oscillator, and the mistuned response does not deviate significantly from the tuned response. As coupling increases, an avenue is created for the blades to communicate vibrational energy, which raises the possiblity of confining energy to a few blades. The mistuned response may then deviate significantly from the tuned response, until further increases in coupling prohibit the confinement of energy, yielding tuned-like response for large coupling values.

Structural coupling of the blades through the disk, which is exemplified by eigenvalue veerings, is only one form of interblade coupling present in in- dustrial rotors. Other forms of interblade coupling include aerodynamic and acoustic coupling. Qualitatively, it is difficult to discern the level of interblade coupling based on eigenvalue curvature due to the fact that natural frequencies only occur at integer numbers of nodal diameters. That is, the modes shapes associated with natural frequencies form standing waves that undergo, over the assembly, integer multiples of $2 \pi$ phase shift. To describe accurately the eigenvalue veerings in Figure 4, a program was written to calculate the frequency corresponding to a traveling wave. The idea is to assume that the interblade phase angle is a continuous variable and solve the eigenvalue problem for a single sector. Thus, boundary degrees of freedom that separate sectors, referred to as side-two nodes in the finite element literature, assume the displacement magnitude associated with the side-one degrees of freedom, except for the addition of an $e^{j \phi}$ phase shift, where the interblade phase angle, $\phi$, is now a continuous variable.

Figure 10 illustrates the traveling wave frequencies for the case study rotor. The percent increase of the largest blade response in the mistuned assembly over the tuned response is indicated at the various veering locations. There are six eigenvalue veerings of interest in Figure 10. Veering 2 is characterized by a large separation distance, reflecting the strong coupling between the first families of disk and blade out-of-plane modes. There is $39 \%, 55 \%$, and $45 \%$ increase in the largest mistuned response over the tuned response for engine order excitations four, five, and six, respectively, in veering 2. Note that the percent increase is largest at the center of the veering.

Interblade coupling is weaker in veering 4 than in veering 2 and thus forced response amplitude increases should be larger for the former. Yet, the percent increases of the mistuned response over the tuned response are equal, since responses can not be calculated in the middle of veering 4 . There is a $23 \%$ and $55 \%$ increase in the mistuned response over the tuned response at engine order excitations 1 and 2 , respectively, in veering 4 . If it were possible to provide noninteger engine order excitation, one speculates that the percent increase in the mistuned response would increase dramatically in veering 4. The importance of where the excitation lies in the veering regions can be further illustrated by examining veerings 5 and 6 . Both veerings exhibit extremely weak interblade coupling, yet engine order excitation 9 falls closer to the center of veering 6 than engine order excitation 5 does to the center of veering 5 . The percent increase of the mistuned 


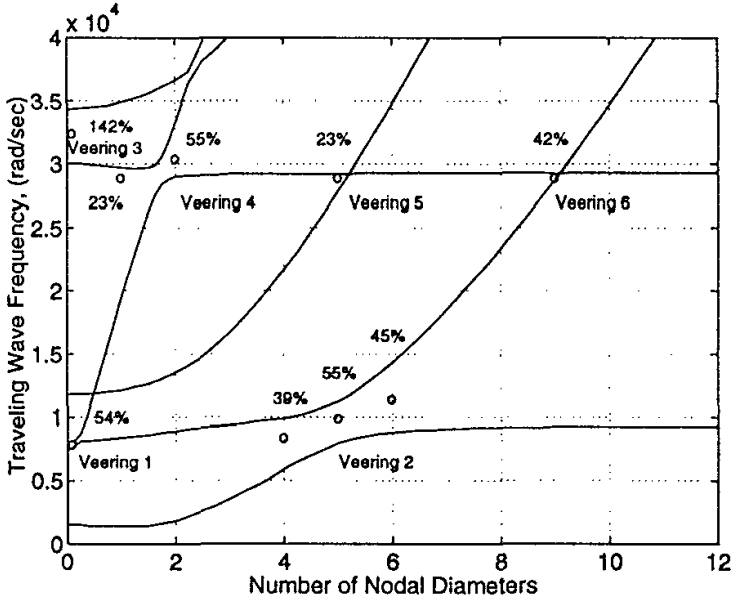

Figure 10: Traveling wave frequencies versus number of nodal diameters as a continuous variable.

system over the tuned system is $42 \%$ in veering 6 , as compared to $23 \%$ for veering 5 . Interblade coupling is larger in veering 1 than in veerings 5 and 6 , and the percent increase, $54 \%$, is higher. Veering 3 , which gives the largest percent increase of the mistuned response over the tuned response, $142 \%$, represents moderately weak interblade coupling.

In summary, there are two competing factors, free mode localization and interblade coupling, which influence response increases due to mistuning. As interblade coupling decreases, an analysis based on mode localization predicts a monotonic increase in response amplitudes. ${ }^{1}$ Yet, if coupling becomes to small, there is no avenue to communicate vibrational energy between blades and mistuned response amplitudes diminish to tuned response predictions. From a design standpoint, the graph of natural frequency versus nodal diameters provides invaluable information about the qualitative behavior of bladed disks. Worst case design and loading conditions are characterized by high modal densities and moderately weak interblade coupling.

4 Numerical Simulations of

Forced Response Statistics Using ROM

As the above free and forced response results illustrate, reduced-order modeling of bladed disks correlates well with much larger finite element models. The technique successfully captures and predicts mistuning effects on response amplitudes-something virtually impossible to achieve for most industrial finite element models. More importantly, as indicated below, reduced-order modeling enables engineers to determine the statistical characteristics of blade forced responses for randomly mistuned bladed disks.

Figure 11 illustrates a Monte Carlo simulation of the statistics of the largest blade response amplitude, at any frequency in the range of 7,500 to $8,500 \mathrm{~Hz}$ (corresponding to the first family of out-ofplane blade modes), and for engine order five excitation. The simulation consists of frequency sweeps of one thousand different mistuning patterns, obtained from a uniform distribution with $5 \%$ standard deviation. Figure 11 required two days of computational time on a $64 \mathrm{Mhz}$ UNIX workstation and was obtained with the standard ROM. The tuned response, which is indicated in Figure 11, represents the optimal distribution of vibratory energy and clearly corresponds to the smallest response amplitude and the minimum stress state. Unfortunately, perfectly tuned bladed disks are not realizable, and the increase in blade response amplitude due to mistuning must be accounted for. Random mistuning must be compensated for by increasing the overall fatigue strength of the blades to meet some statistically determined stress level. Alternatively, if feasible, the designer could choose a deliberate mistuning pattern that minimizes the increase in mistuned vibratory stresses. In both instances, reduced-order modeling can aid the designer in capturing mistuning effects.

In Fig. 11, the 95th percentile of the largest blade response amplitude (the amplitude such that $95 \%$ of all mistuned rotors feature a smaller largest amplitude) is seen to correspond to a $47 \%$ increase over the tuned system resonant response amplitude. The corresponding 95th percentile principal stresses are $\sigma_{1}=526.2, \sigma_{2}=-1.5$, and $\sigma_{3}=-88.9 \mathrm{MPa}$. Recall that the tuned principal stresses are $\sigma_{1}=357.9$, $\sigma_{2}=-1.0$, and $\sigma_{3}=-59.3 \mathrm{MPa}$. Stresses for the 95 th percentile of the largest blade response amplitude are thus as much as $47 \%$ higher than the corresponding tuned stresses. If the current design is based on tuned stress magnitudes, fatigue properties of the blades should be increased to compensate for the random nature of mistuning (assuming it is acceptable that $5 \%$ of bladed disks do not meet this condition). Alternatively, Table 5 lists a 5 th percentile mistuning pattern in which the largest mistuned vibratory stresses are $\sigma_{1}=423.7, \sigma_{2}=$ -1.2 , and $\sigma_{3}=-70.9 \mathrm{MPa}$. This deliberate mistuning pattern limits the principal stress increase over tuned levels to only $18 \%$. The choice of increasing the fatigue strength to some statistically determined amplitude or of deliberately mistuning the system is up to the designer. But, it should be pointed out that a specific mistuning pattern may not reduce the vibratory stresses at other frequency regions of 


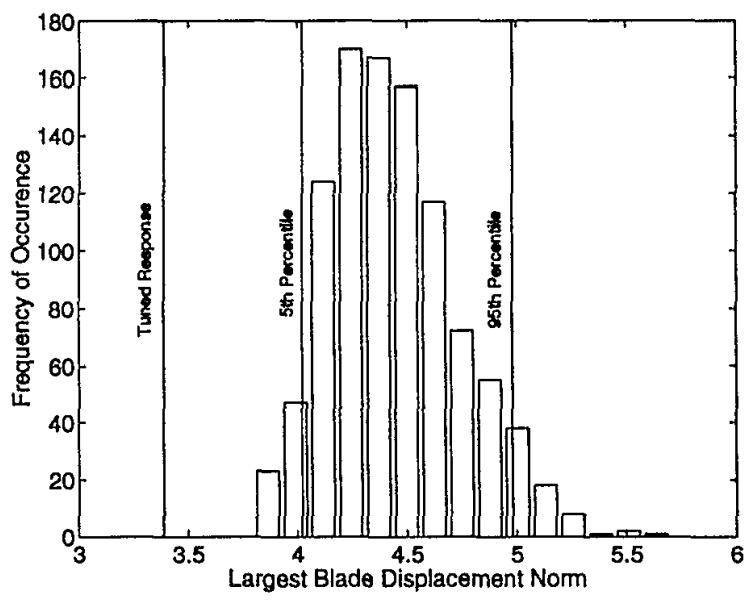

Figure 11: Histogram of the largest blade response amplitude at any frequency, for out-of-plane excitation with $C=5$. Obtained by Monte Carlo simulation of 1,000 different mistuned systems with uniform distributions of $5 \%$ standard deviation.

\begin{tabular}{||c|c||}
\hline \hline Blade Number & Mistuning $\delta_{i}^{\sigma}$ \\
\hline 1 & -0.044449 \\
2 & -0.033217 \\
3 & -0.036770 \\
4 & 0.041214 \\
5 & -0.059288 \\
6 & 0.077369 \\
7 & -0.017369 \\
8 & 0.009893 \\
9 & 0.039600 \\
10 & 0.002804 \\
11 & -0.014880 \\
12 & 0.077372 \\
13 & -0.020565 \\
14 & 0.073124 \\
15 & 0.021230 \\
16 & -0.059587 \\
17 & -0.021516 \\
18 & -0.028875 \\
19 & -0.041692 \\
20 & -0.059717 \\
21 & 0.066580 \\
22 & -0.010611 \\
23 & 0.085981 \\
24 & 0.004279 \\
\hline
\end{tabular}

Table 5: Random mistuning distribution that limits the largest vibratory stress level (corresponding to the 5 th percentile of the largest reponse amplitude statistics). operation or in other modes of vibration. In fact, a specific mistuning pattern may result in larger increases in vibratory stresses in other frequency regions. Statistically determining the maximum vibratory stress levels and increasing the fatigue strength of the individual blades is a more robust design strategy if the operational range of rotor encompasses several modes of vibration.

\section{Conclusions}

In this paper, the ROM free response formulation presented by Ottarsson et al. ${ }^{3}$ was successfully extended to the forced response case. The ROM forcing vector was expressed in terms of single sector quantities, which minimizes computer memory and computational costs.

Responses from reduced-order and finite element models were compared for a case study rotor. Both resonant displacement amplitudes and principal stresses from the ROM were within $10 \%$ of the finite element model. The key distinction between the two solutions is the computational savings associated with the ROM. The mistuned finite element model had 8,640 degrees of freedom, whereas the mistuned ROM had 216 degrees of freedom. Once satisfied that the ROM correlated well to the finite element model of the rotor, the ROM was used to determine the statistical characteristics of forced responses for randomly mistuned bladed disks. A Monte Carlo simulation of one thousand different mistuning patterns found that $95 \%$ of all mistuned rotors featured response amplitude and principal stress increases that were at most $47 \%$ over their tuned counterparts. The ROM was further used to determine a deliberate mistuning pattern that limited the increase in response amplitudes and principal stresses due to mistuning to less than $18 \%$.

\section{References}

1 S. T. Wei and C. Pierre. "Localization Phenomena in Mistuned Assemblies with Cyclic Symmetry, Part I: Free Vibrations". ASME Journal of Vibration, Acoustics, Stress, and Reliability in Design, 110(4):429-438, 1988.

${ }^{2}$ M. J. Kruse and Pierre C. "Dynamic Response of an Industrial Turbomachinery Rotor". In Proceedings of the 32nd AIAA/ASME/SAE/ASEE Joint Propulsion Conference and Exhibit, Lake Buena Vista, Florida, July 1996.

${ }^{3}$ G. S. Ottarsson, M. P. Castanier, and C. Pierre. "A Reduced-Order Modeling Technique for Mis- 
tuned Bladed Disks". In Proceedings of the 35th AIAA/ASME/ASCE/AHS/ASC Strutures, Structural Dynamics, and Materials Conference., 1994, Also, ASME Journal of Vibration and Acoustics, in print.

${ }^{4}$ J.A. Fabunmi. "Forced Vibration of a Single Stage Axial Compressor Rotor". ASME Journal of Engineering for Power, 102(2):322-329, 1980.

5 J. H. Griffin and T. M. Hoosac. "Model Development and Statistical Investigation of Turbine Blade Mistuning". ASME Journal of Vibration, Acoustics, Stress, and Reliability in Design, 106:204-210, 1984.

6 P. J. Davies. "Circulant Matrices". WileyIntersciences, 1979.

7 Pierre C. "Mode Localization and Eigenvalue Loci Veering Phenomena in Disordered Structures". Journal of Sound and Vibration, 126(3):485-502, 1988.

${ }^{8}$ S. T. Wei and C. Pierre. "Localization Phenomena in Mistuned Assemblies with Cyclic Symmetry, Part II: Forced Vibrations". ASME Journal of Vibration, Acoustics, Stress, and Reliability in Design, 110(4):439-449, 1988.

${ }^{9}$ G. S. Ottarsson and C. Pierre. "On the Effects of Interblade Coupling on the Statistics of Maximum Forced Response Amplitudes in Mistuned Bladed Disks". In Proceedings of the 36th AIAA/ASME Strutures, Structural Dynamics, and Materials Conference, New Orleans, Louisiana, 1995.

\section{Appendix A}

The general form of a square circulant matrix is

$\mathrm{C}=\operatorname{circ}\left(\mathbf{c}_{1}, \mathbf{c}_{2}, \ldots, \mathbf{c}_{N}\right)=\left[\begin{array}{cccr}c_{1} & c_{2} & \ldots & c_{N} \\ c_{N} & c_{1} & \ldots & c_{N-1} \\ \vdots & \vdots & \ddots & \vdots \\ c_{2} & c_{3} & \ldots & c_{1}\end{array}\right]$

Circulant matrices of order $N$ posses $N$ independent eigenvectors. Furthermore, all circulant matrices share the same set of eigenvectors that make up the Fourier matrix:

$$
\begin{gathered}
\mathrm{E}=\left(e_{k i}\right) \quad k, i=1, \ldots, N \\
e_{k i}=\frac{1}{\sqrt{N}} e^{\frac{j 2 \pi(i-1)(k-1)}{N}} \quad k, i=1, \ldots, N
\end{gathered}
$$

The real-valued form of Eq. (A.2) is:

$$
\underline{e}_{k i}=\left\{\begin{array}{cll}
\frac{1}{\sqrt{N}} & \text { if } & i=1 \\
\sqrt{\frac{2}{N}} \cos \left(\frac{2 \pi(i-1)(k-1)}{N}\right) & \text { if } & 1<i<P \\
\frac{-1^{k-1}}{\sqrt{N}} & \text { if } & i=P, \\
\sqrt{\frac{2}{N}} \sin \left(\frac{2 \pi(i-1)(k-1)}{N}\right) & \text { if } & P<i \leq N \\
k=1, \ldots, N &
\end{array}\right.
$$

The Kronecker product of two matrices is

$$
\mathbf{C}=\mathbf{A} \otimes \mathbf{B}=\left[\begin{array}{cllr}
a_{11} \mathbf{B} & a_{12} \mathbf{B} & \ldots & a_{1 N} \mathbf{B} \\
a_{21} \mathbf{B} & a_{22} \mathbf{B} & \ldots & a_{2 N} \mathbf{B} \\
\vdots & \vdots & \ddots & \vdots \\
a_{N 1} \mathbf{B} & a_{N 2} \mathbf{B} & \ldots & a_{N N} \mathbf{B}
\end{array}\right]
$$

A useful property of the Kronecker product is

$$
(A \otimes B)(C \otimes D)=(A C) \otimes(B D)
$$

\section{Appendix B: Nomenclature}

(Matrix dimensions in parenthesis)

$C \quad$ Engine order excitation

$E_{i} \quad$ Young's modulus of blade $i$

$E_{0} \quad$ Young's modulus for a tuned blade

$G_{S t r u c}$ Structural damping coefficient

$j \quad$ Imaginary number $\equiv \sqrt{-1}$

$m \quad$ Number of modes

$m_{b} \quad$ Number of cantilever blade modes

$m_{d} \quad$ Number of disk-induced static modes

$n \quad$ Harmonic number

$n_{F E} \quad$ Number of blade finite element degrees of freedom per sector

$N \quad$ Number of blades

$N_{d i a} \quad$ Number of nodal diameters

$P \quad$ Maximum number of harmonics, or nodal diameters, $P=\operatorname{int}\left[\frac{N}{2}\right]$

$\delta_{i} \quad i$ th blade stiffness mistuning

$\delta T \quad$ Variation of kinetic energy

$\delta u \quad$ Virtual displacement

$\delta U \quad$ Variation of strain energy

$\delta W_{\text {ext }}$ External virtual work

$\zeta \quad$ Viscous damping ratio

$\phi_{i} \quad$ Interblade phase angle, $i=1, \ldots, P$

$\omega \quad$ Excitation frequency

$\otimes \quad$ Kronecker product (See Appendix A)

$a_{\mathbf{n}} \quad$ Vector of generalized coordinates corresponding to the $n$ nodal diameter disk modes $\left(m_{d}, 1\right)$ 
a Vector of generalized coordinates for all disk modes, $\mathbf{a}=\left[\mathbf{a}_{0}^{\mathbf{T}}, \mathbf{a}_{\mathbf{1}}^{\mathbf{T}}, \ldots, \mathbf{a}_{\mathbf{P}}^{\mathbf{T}}\right]^{\mathbf{T}}$

$\mathbf{b}_{\mathbf{i}} \quad$ Vector of generalized coordinates corresponding to the $i$ th blade's cantilevered modes $\left(m_{b}, 1\right)$

b Vector of generalized coordinates for all $N$ blades, $\mathbf{b}=\left[\mathbf{b}_{\mathbf{1}}^{\mathbf{T}}, \mathbf{b}_{\mathbf{2}}^{\mathbf{T}}, \ldots, \mathbf{b}_{\mathbf{N}}^{\mathbf{T}}\right]^{\mathbf{T}}\left(m_{b} N, 1\right)$

E Fourier matrix

$e_{\mathbf{n}} \quad n$th column of the Fourier matrix

$\underline{\mathbf{e}}_{\mathbf{n}}^{\mathbf{c}} \quad n$th harmonic cosine vector from the real form of the Fourier matrix

$\mathrm{e}_{\mathrm{n}}^{\mathrm{s}} \quad n$th harmonic sine vector from the real form of the Fourier matrix

$\tilde{\mathbf{f}} \quad$ Force vector on a single blade $\left(n_{F E}, 1\right)$

F Forcing vector on the entire assembly of blades $\left(n_{F E} N, 1\right)$

$\tilde{\mathbf{K}}_{\mathrm{b}} \quad$ Finite element stiffness matrix of a free blade $\left(n_{F E}, n_{F E}\right)$

$K_{b} \quad$ Blade portion of the stiffness matrix of the entire assembly $\left(n_{F E} N, n_{F E} N\right)$

$\hat{\mathbf{K}}_{\mathrm{d}} \quad$ Modal stiffness matrix of the entire disk $\left(m_{d} N, m_{d} N\right)$

$\tilde{\mathbf{M}}_{\mathbf{b}} \quad$ Finite element mass matrix of a single blade $\left(n_{F E}, n_{F E}\right)$

$\mathrm{M}_{\mathrm{b}} \quad$ Blade portion of the mass matrix of the entire assembly $\left(n_{F E} N, n_{F E} N\right)$

$\tilde{\mathbf{u}}_{\mathbf{i}}^{\mathbf{b}} \quad$ ith cantilever blade mode for one blade $\left(n_{F E}, 1\right)$

$\tilde{U}^{\mathbf{b}} \quad$ Matrix of cantilever blade modes for one blade $\left(n_{F E}, m_{b}\right)$

$U^{b}$ Cantilever blade modes for the entire $\operatorname{assembly}\left(n_{F E} N, m_{b} N\right)$

$\tilde{\mathbf{u}}_{\mathbf{n}, \mathbf{m}}^{\mathbf{d}}, \tilde{\mathbf{u}}_{\mathbf{n}, \mathbf{m}}^{\mathbf{d}^{*}}$ Complex conjugate pair of diskinduced modes, $n$th harmonic, $m$ th mode $\left(n_{F E}, 1\right)$

$\tilde{\mathbf{u}}_{\mathbf{n}, \mathbf{m}}^{\mathbf{c}} \quad$ Real part of $\tilde{\mathbf{u}}_{\mathbf{n}, \mathbf{m}}^{\mathbf{d}}$

$\tilde{\mathbf{u}}_{\mathbf{n}, \mathbf{m}}^{\mathbf{s}}$ Imaginary part of $\tilde{\mathbf{u}}_{\mathbf{n}, \mathbf{m}}^{\mathbf{d}}$

$\tilde{\mathbf{U}}_{\mathbf{n}}^{\mathbf{d}} \quad$ Matrix of $n$th harmonic disk-induced modes for a single sector $\left(n_{F E}, m_{d}\right)$

$\mathbf{U}_{\mathbf{n}, \mathbf{m}}^{\mathbf{d}}, \mathbf{U}_{\mathbf{n}, \mathbf{m}}^{\mathbf{d}^{*}}$ Complex conjugate pair of diskinduced modes for the entire assembly, $n$th harmonic, $m$ th mode $\left(n_{F E} N, 1\right)$

$\mathrm{U}_{\mathbf{n}}^{d}$ Matrix of $n$th harmonic diskinduced modes for the entire assembly $\left(n_{F E} N, m_{d}\right)$

$\mathbf{U}^{\mathrm{d}} \quad$ Matrix of disk-induced modes for the entire assembly $\left(n_{F E} N, m_{d} N\right)$ 